\title{
An Overview of AUV Algorithms Research and Testbed at the University of Michigan
}

\author{
Ryan M. Eustice*, Hunter C. Brown*, Ayoung Kim ${ }^{\dagger}$ \\ *Department of Naval Architecture \& Marine Engineering \\ ${ }^{\dagger}$ Department of Mechanical Engineering \\ University of Michigan \\ Ann Arbor, Michigan 48109-2145 \\ email:\{eustice, hcbrown, ayoungk\}@umich.edu
}

\begin{abstract}
This paper provides a general overview of the autonomous underwater vehicle (AUV) research projects being pursued within the Perceptual Robotics Laboratory (PeRL) at the University of Michigan. Founded in 2007, PeRL's research thrust is centered around improving AUV autonomy via algorithmic advancements in sensor-driven perceptual feedback for environmentally-based real-time mapping, navigation, and control. In this paper we discuss our three major research areas of: (1) real-time visual simultaneous localization and mapping (SLAM); (2) cooperative multi-vehicle navigation; and (3) perception-driven control. Pursuant to these research objectives, PeRL has acquired and significantly modified two commercial off-the-shelf (COTS) Ocean-Server Technology, Inc. Iver2 AUV platforms to serve as a real-world engineering testbed for algorithm development and validation. Details of the design modification, and related research enabled by this integration effort, are discussed herein.
\end{abstract}

\section{INTRODUCTION}

The Perceptual Robotics Laboratory (PeRL) at the University of Michigan (UMich) is actively involved in three major research efforts: real-time vision-based simultaneous localization and mapping (SLAM), heterogeneous multi-vehicle cooperative navigation, and perception-driven control. The laboratory chose to purchase two commercial off-the-shelf (COTS) Ocean-Server Technology autonomous underwater vehicles (AUVs) to support these research goals, and upgraded the vehicles with additional perceptual and navigation sensors to enable this research.

\section{A. Real-Time Visual SLAM}

The first of the three PeRL research domains, real-time vision-based SLAM algorithms [1]-[4], has direct application to ship-hull inspection [5] and deep sea archaeological missions [6]. Present day means for ship hull and port facility inspection require either putting divers in the water or piloting a remotely operated vehicle (ROV) over the area of interestboth of which are manpower intensive and generally cannot guarantee $100 \%$ survey coverage. Automating this task, however, is challenging and compounded by the fact that areas around ships in berth are severely confined, cluttered, and complex sensing environments (e.g., acoustically, optically, magnetically). Current tethered robotic inspection systems present issues of snagging, maneuver degradation, and tether management, all of which make maneuvering around the ship at pier difficult. Moreover, current robotic inspection methods require human in-the-loop intervention for both sensory interpretation and control (piloting). Navigation feedback in these scenarios is typically performed using acoustic transponderbased time-of-flight ranging [7], [8]. This necessitates setup and calibration of the associated acoustic-beacon navigation infrastructure, and therefore vitiates our ability to rapidly and repeatably inspect multiple underwater structures.

In light of this, there exists a need to automate this task through the use of untethered robotic vehicles. To do so with AUVs requires overcoming several present-day science and technology challenges inherent to the inspection task. For example, areas around ships in berth are severely confined, cluttered, and complex sensing environments (e.g., rudders, screws). This necessitates the need for advanced navigation and localization systems that can work in confined, magnetically noisy spaces. In addition, to ensure 100\% survey coverage of ship hulls, pier structures, and pilings requires technological advances in our understanding of autonomous environmental perception and control. The underlying algorithm should facilitate in-situ sensor-reactive navigation and mapping in these environments while accommodating mapbased learning through time via revisited exploration (a prerequisite for hull change detection). Moreover, the increased diversity of threat objects and associated potential for more false alarms due to a cluttered environment necessitates that fusion take place from multiple types of sensors for robustness and redundancy. In combination, all of these individual challenges/requirements, together, suggest that a feature-based navigation and mapping strategy approach would accommodate the needs of autonomous automated search and inspection by AUVs.

The technical objective of this work is to develop an optical/acoustic real-time feature-based navigation (FBN) capability for explosive ordnance disposal (EOD) autonomous ship-hull inspection. Fig. 1 depicts core elements of the overall FBN methodology, called visually augmented navigation (VAN). The VAN framework uses visual perception to augment the onboard dead-reckon navigation capabilities of the unmanned underwater vehicle (UUV). VAN uses a posegraph SLAM framework [3], [4], [9] to incorporate pairwise constraints from overlapping sensor imagery. These constraints 


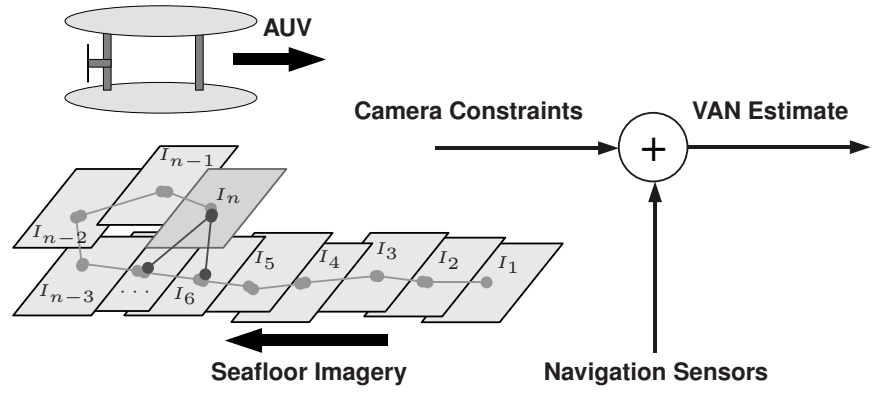

(a) VAN methodology.

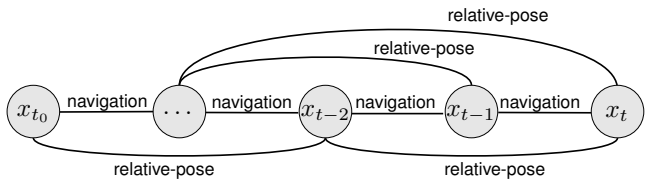

(b) Pose-graph

Fig. 1. The foundation of visually augmented navigation (VAN) is the fusion of "zero-drift" camera measurements with dead-reckoned vehicle navigation data to produce a bounded error position estimate. These constraints are fused with onboard navigation sensor data in a view-based stochastic map framework; the model is comprised of a pose-graph where the nodes correspond to historical robot poses and the edges represent either navigation (first-order Markov) or camera (non-Markov) constraints.

form edges in the pose-graph and constrain the vehicle position estimate to bounded precision. This type of view-based approach is ideally suited to the ship-hull inspection task since the goal is to provide $100 \%$ survey coverage of the hull with minimal trajectory redundancy.

We are currently in Year 1 of a three year project to develop a real-time feature-based navigation system. Years 1 and 2 are focused on developing the overall mapping framework using vision as the main perceptual sensor. Year 3 of the project will investigate transitioning the VAN framework to sonar-based perception, which will yield a larger standoff range sensing capability in turbid water. The transition to sonar perception will require developing an appropriate sonar registration engine so that overlapping sonar images can be registered to extract pose-constraints similar to the optical registration engine of [4]. This work will explore the adaptation of feature extraction and description techniques developed within the computer vision community to the physics constraints of sonar imaging.

\section{B. Cooperative Navigation}

In addition to real-time visual SLAM, PeRL is working toward cooperative multi-vehicle missions for large-area survey. Multi-vehicle cooperative navigation offers promise of efficient exploration by groups of mobile robots working together to pool their mapping capability. Most prior research in the SLAM community has focused on the case of singleagent mapping and exploration. While these techniques can often be extended to a centralized multi-agent framework [10] (provided that there are no communication bandwidth restrictions), the extension of single-agent techniques to a decentralized multi-vehicle SLAM framework is often not obvious nor appropriate. Much of the previous research in the area of distributed multi-vehicle SLAM has focused primarily on terrestrial (i.e., land and aerial) applications [11][15], where high-bandwidth radio communication is possible; however, underwater communication bandwidth is distinctly limited from that on land [16].

The speed of sound in water is $1500 \mathrm{~m} / \mathrm{s}$, which is $1 / 200,000$ that of the speed of light! Moreover, it requires on the order of 100 times more power to transmit than it does to receive, making acoustic transmission and reception asymmetrical for medium access (MAC) schemes [16]. Halfduplex time division multiple access (TDMA) networks are usual, with typical acoustic-modem data rates ranging from $5 \mathrm{kbits} / \mathrm{s}$ at a range of $2 \mathrm{~km}$ (considered a high rate), to as little as $80 \mathrm{bits} / \mathrm{s}$ (a low rate). The low acoustic data rates are not simply a limitation of current technology-the theoretical performance limit for underwater acoustic communications is $40 \mathrm{~km} \cdot \mathrm{kbps}$ (i.e., a max theoretical data rate of $20 \mathrm{kbps}$ at a range of $2 \mathrm{~km}$, for example) [16]. Therefore, any type of multi-vehicle SLAM framework must adhere to the physical bandwidth limitations of the underwater domain.

In previous work, Eustice et al. developed a synchronousclock acoustic modem-based navigation system capable of supporting multi-vehicle ranging [9], [17]. The system consisted of a WHOI Micro-Modem [18], [19] (an underwater acoustic modem developed by Woods Hole Oceanographic Institution (WHOI)) and a low-power stable clock board. This system can be used as a synchronous-transmission communication/navigation system wherein data packets can encode time of origin information as well as local ephemeris data (e.g., $x, y, z$ positional data and error metric). This allows for the direct measurement of inter-vehicle one-way travel time (OWTT) time-of-flight ranging. The advantage of a OWTT ranging methodology is that all passively receiving nodes within listening range are able to decode and measure the inter-vehicle range to the broadcasting node.

PeRL is currently investigating probabilistic fusion methods for a OWTT multi-vehicle framework that scales across a distributed network of multiple agents who have limited communication bandwidth in a non-fully-connected network topology. The proposed acoustic-modem augmented navigation framework will exploit inter-vehicle OWTT ranging to supplement perceptual SLAM localization while reducing the need for state communication. The goal is to distribute state estimation between the vehicles in a coordinated fashionallowing for navigation impoverished vehicles (e.g., no inertial measurement unit (IMU) or Doppler velocity log (DVL)) to share from positional accuracies of better equipped vehicles (e.g., those with DVL bottom-lock).

For example, a near-seafloor VAN [4] enabled vehicle could perform highly detailed inspection over small regions, while a mid-water column vehicle enabled with side-scan sonar could traverse larger regions to identify specific sites of interest. As the VAN vehicle's state estimation improves over time, distributing this knowledge to the other vehicle could 


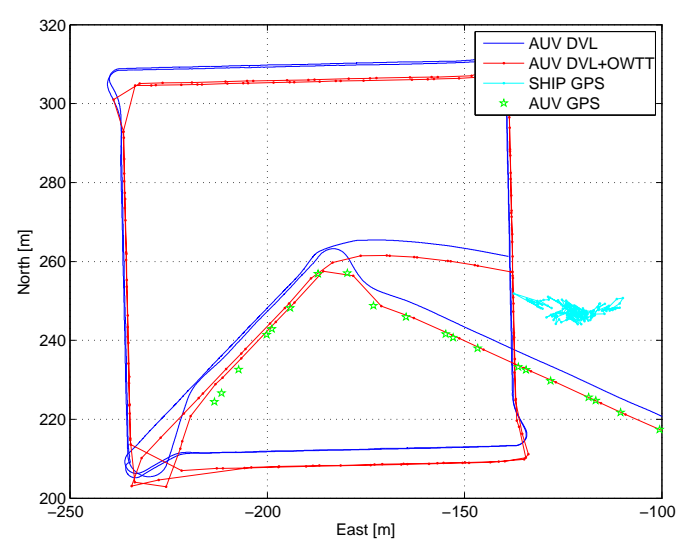

Fig. 2. Preliminary OWTT results as reported in [9], [17] for a two node network consisting of an AUV and a surface ship. Shown in blue is the raw dead-reckoned AUV trajectory; in cyan is the GPS-derived ship position; in red is the OWTT fused AUV trajectory; and in green is the (on-surface) GPSmeasured AUV position, which serves as an independent ground-truth.

refine the non-VAN vehicles' state estimate. This coordinated effort would result in faster overall survey times and more attention to regions of interest, rather than using the full VAN capabilities over feature-barren tracts of seafloor.

Fig. 2 depicts preliminary results, reported in [9], [17], demonstrating the OWTT proof of concept. Here, a global positioning system (GPS)-equipped surface ship navigationally aided a submerged AUV by broadcasting ship GPS position to the network while the AUV measured its range to the ship via the OWTTs.

\section{Perception-Driven Control}

Another research focus is in the domain of perceptiondriven control. Algorithms are under development to enable a vehicle to respond to the environment by autonomously selecting alternative search patterns based on perceived feature distributions in the observed data. This creates improvements in productivity by eliminating long surveys of feature-poor areas and instead allows more bottom-time spent over actual targets. A seafloor survey vehicle, for example, may drive into an area devoid of features during a mission. Instead of continuing to search the featureless space, where there is little return on investment from the expense of running a vision system, the vehicle would return to a previously known feature rich area and begin searching in another direction. The PeRL group is currently working on algorithms to assist in the decision making process of when to revisit known landmarks versus continuing new exploration.

\section{Testbed Overview}

To pursue PeRL's research objectives, two commercial Ocean-Server Iver2 AUV systems were purchased and modified to serve as real-world testbed platforms for SLAM research at UMich. Although several other vehicle platforms currently include stereo-vision systems and DVL sensors, the Iver2 (Fig. 3) was selected as a testbed development platform due to its ability to be transported in a personal vehicle and launched by a single user. The vehicles, as shipped, are rated to a depth of $100 \mathrm{~m}$, have a maximum survey speed of approximately $2 \mathrm{~m} / \mathrm{s}$ ( 4 knots), and weigh $\sim 30 \mathrm{~kg}$ allowing for transport by two people [20].

Since the COTS Iver2 vehicle does not come equipped with camera or DVL sensing, sensor upgrades were required to enable the original vehicle to perform SLAM and coordinated multi-AUV missions. PeRL upgraded the vehicles with additional navigation and perceptual sensors (detailed in Fig. 4 and Table I) including 12-bit stereo down-looking Prosilica cameras, a Teledyne $600 \mathrm{kHz}$ RDI Explorer DVL for 3-axis bottom-lock velocity measurements, a KVH single-axis fiberoptic gyroscope for yaw rate, and a WHOI Micromodem for communication, along with other sensor packages discussed forthwith. To accommodate the additional sensor payload, a new Delrin nose cone was designed and fabricated (Fig. 6). Additional 32-bit embedded PC104 CPU hardware was added for data-logging, real-time control, and in-situ real-time SLAM algorithm testing and validation. Details of the design modification are discussed herein.

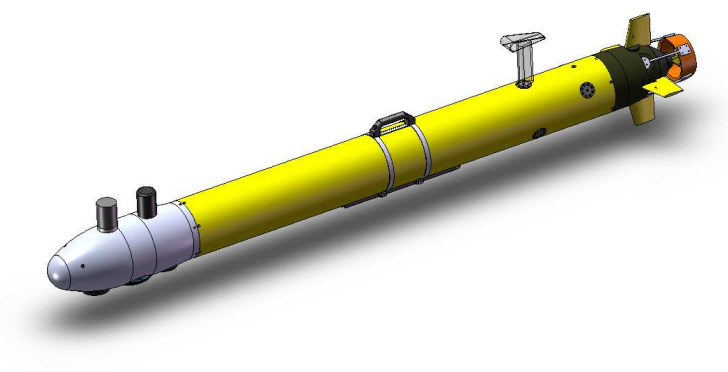

Fig. 3. Modified OceanServer Iver2.

\section{A. Mechanical/Electrical Design and Integration}

The design goals during the integration phase of vehicle development consisted of minimizing hydrodynamic drag, maintaining neutral buoyancy, and maximizing sensor payload capacity within the pressure hull. These requirements were achieved through the use of lightweight materials such as acrylonitrile butadiene styrene (ABS), Delrin, and aluminum, and careful center of buoyancy and center of mass computations. The entire vehicle was modeled using Solidworks solid modeling software and extensive use of these computer aided design (CAD) models provided optimal arrangements of internal components prior to actual installation (Fig. 5).

The addition of a redesigned SLAM nose cone and sensor payload shifted both the original center of buoyancy and center of gravity. New positions were estimated using the CAD models and refined during ballast tests at the UMich Marine Hydrodynamics Laboratory (MHL). The vehicle is ballasted to achieve $\sim 0.11 \mathrm{~kg}(0.25 \mathrm{lbs})$ reserve buoyancy for emergency situations when the vehicle must surface without power. Vehicle trim is set neutral to achieve passive stability and to optimize both diving and surfacing operations. 


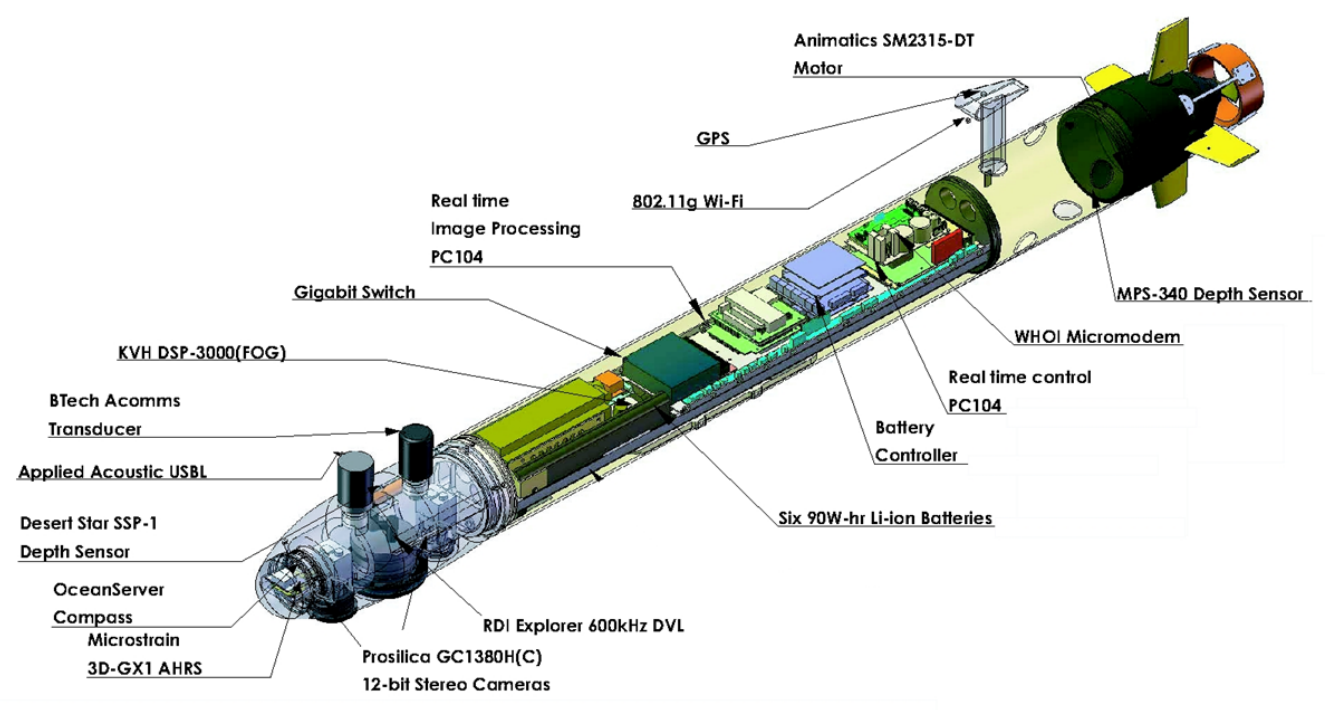

Fig. 4. PeRL modified Iver2 sensor and computing layout.

TABLE I

INTEGRATED SENSORS ON THE PERL VEHICLES.

\begin{tabular}{|c|c|c|c|c|c|}
\hline IVER2 INSTRUMENTS & VARIABLE & UPDATE RATE & PRECISION & RANGE & DRIFT \\
\hline OceanServer OS5000 Compass & attitude & $0.01-20 \mathrm{~Hz}$ & $1-3^{\circ}$ (Heading), $2^{\circ}$ (Roll, Pitch) & $360^{\circ}$ & - \\
\hline $\begin{array}{l}\text { Measurement Specialties Pressure } \\
\text { Sensor MSP-340 }\end{array}$ & depth & - & $<1 \%$ of FS & $0-300 \mathrm{psi}$ & - \\
\hline $\begin{array}{lll}\text { Imagenex Sidescan Sonar (Dual } \\
\text { Freq.) }\end{array}$ & - & 330 or $800 \mathrm{kHz}$ & - & $15-120 \mathrm{~m}$ & - \\
\hline USGlobalSat EM-406a GPS & XYZ position & $1 \mathrm{~Hz}$ & $5-10 \mathrm{~m}$ & - & - \\
\hline NEW INSTRUMENTS & VARIABLE & UPDATE RATE & PRECISION & RANGE & DRIFT \\
\hline $\begin{array}{lll}\begin{array}{l}\text { Prosilica } \\
\text { (down-looking stereo-pair) }\end{array} & \text { Camera } \\
\end{array}$ & gray/color image & $1-5$ fps & $1360 \times 1024$ pixels; 12 -bit depth & - & - \\
\hline $\begin{array}{l}\text { Teledyne RDI } 600 \mathrm{kHz} \text { Explorer } \\
\text { DVL }\end{array}$ & body velocity & $7 \mathrm{~Hz}$ & $1.2-6 \mathrm{~cm} / \mathrm{s}(@ 1 \mathrm{~m} / \mathrm{s})$ & $0.7-65 \mathrm{~m}$ & - \\
\hline KVH DSP-3000 single-axis FOG & yaw rate & $100 \mathrm{~Hz}$ & $1-6^{\circ} / \mathrm{h}$ & $\pm 375^{\circ} / \mathrm{s}$ & $4^{\circ} / \mathrm{h} / \sqrt{\mathrm{Hz}}$ \\
\hline $\begin{array}{l}\text { Desert Star SSP-1 300PSIG Digital } \\
\text { Pressure Transducer }\end{array}$ & depth & $.0625-4 \mathrm{~Hz}$ & $0.2 \%$ of FS & $0-300 \mathrm{psi}$ & - \\
\hline Applied Acoustics USBL & XYZ position & $1.0-10.0 \mathrm{~Hz}$ & $\pm 0.1 \mathrm{~m}$ (Slant Range) & $1000 \mathrm{~m}$ & - \\
\hline$\frac{\text { OWTT }^{*} \text { Nav (Modem+PPS) }}{\text {-WHOI Micromodem }}$ & $\begin{array}{c}\text { slant range } \\
\text { communication }\end{array}$ & Varies & 18.75 cm (@1500 m/s) & Varies & $<1.5 \mathrm{~m}$ in $14 \mathrm{~h}$ \\
\hline -Seascan SISMTB v.4 PPS Clock & time & $1 \mathrm{~Hz}$ & $1 \mu \mathrm{s}$ & - & $1 \mathrm{~ms} / 14 \mathrm{~h}$ \\
\hline Microstrain 3D-GX1 AHRS & attitude, body rates & $1.0-100 \mathrm{~Hz}$ & $\pm 0.5^{\circ}$ & $\pm 180,180,90$ & - \\
\hline
\end{tabular}

* One-Way-Travel-Time (OWTT)

In determining the location and orientation of each component within the vehicle body, heat dissipation and interference in-between sensors were considered as the critical constraints after spatial constraints were satisfied. Due to the high density of sensors and other devices in the pressure housing, the components with the highest heat radiation, such as computers and dc-dc converters, are placed in direct contact with the aluminum chassis to allow better heat dissipation. Also, sensors that are prone to electrical noise from surrounding electronics are spatially separated in the layout (e.g. the MEMS Microstrain 3D-GX1 is located in the nose cone tip, the furthest point from motor and battery pack influence).

Electrically, the vehicle is powered by a $590 \mathrm{Whr} \mathrm{Li}$ ion battery pack made up of six 95 Whr laptop batteries managed by an Ocean-Server Intelligent Battery and Power System module. The added sensors and PC104 computing draw $53 \mathrm{~W}$. This load is in addition to the original $9 \mathrm{~W}$ nominal vehicle hotel load and $110 \mathrm{~W}$ propulsion load of the stock Iver2 resulting in a combined maximum total power draw of $\sim 172 \mathrm{~W}$ (this assumes full hotel load and the motor at full power.) Future plans include integrating a seventh battery to achieve $665 \mathrm{Whr}$ for an estimated run time of 3.8 hours at maximum speed (5 hours or more at $75 \mathrm{~W}(2 \mathrm{kt})$ propulsion speed).

\section{B. SLAM Nose cone}

In order to support the real-time VAN objectives of PeRL, a down-looking stereo-vision system was added to the Iver2 


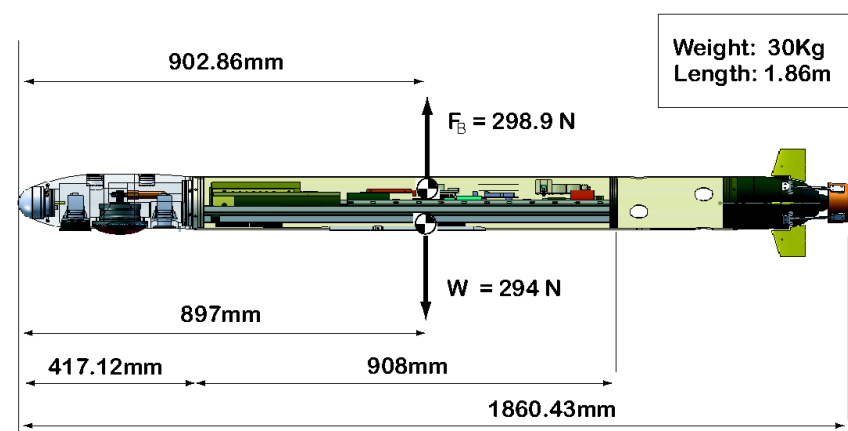

Fig. 5. Mechanical layout.

vehicles. Stereo vision allows scale information to be extracted from pairwise image registration thereby improving state estimation by observing velocity scale error in DVL measurements. A new nose cone was designed and fabricated to house both the two-camera vision system and the DVL transducer.

The UMich custom-design nose cone (Fig. 6) was fabricated from Acetron GP (Delrin) due to the material's high tensile strength, scratch resistance, fatigue endurance, low friction, and low water absorption. Threaded inserts are installed in the nose cone to prevent stripped threads and stainless fasteners with a polytetrafluoroethene (PTFE) paste (to prevent corrosion issues) are used in all locations.

The designed working depth of the nose cone is $100 \mathrm{~m}$ (to match the full rating of the Iver2). Calculations were performed according to ASME Section VIII Boiler and Pressure Vessel Code to verify wall thickness in each of the nose cone sections. A minimum factor of safety of at least 2.64 was attained for all sections of the nose cone. Pressure tests, conducted at Woods Hole Oceanographic Institution, demonstrated the structural integrity of the nose cone to $240 \mathrm{~m}$ water depth. Three short duration dives of 12 minutes each were made to $360 \mathrm{psi}$ and one long duration dive of five hours was made to $360 \mathrm{psi}$.

The Teledyne-RDI $600 \mathrm{kHz}$ Explorer DVL (Fig. 6) is integrated into the nose cone using fasteners to attach the DVL head to threaded inserts in the nose cone material. The internal cavity space precludes the use of the recommended clamp attachment scheme. Self-sealing fasteners are used to eliminate a fluid path through the mounting holes of the DVL to the interior of the nose cone.

Two nose cone plugs were designed for camera integration that include a sapphire window and two mounting brackets each (Fig. 7). The synthetic sapphire window was chosen due to the high scratch resistance and superior tensile strength of sapphire versus plastic or glass materials. The mounting brackets were designed in CAD and printed in ABS plastic using a Dimension FDM Elite rapid prototype machine. Static face and edge o-ring seals prevent water ingress through the plug around the sapphire window.

A Desert Star SSP-1 pressure transducer is mounted to an internal face of the nose cone and is exposed to the ambient
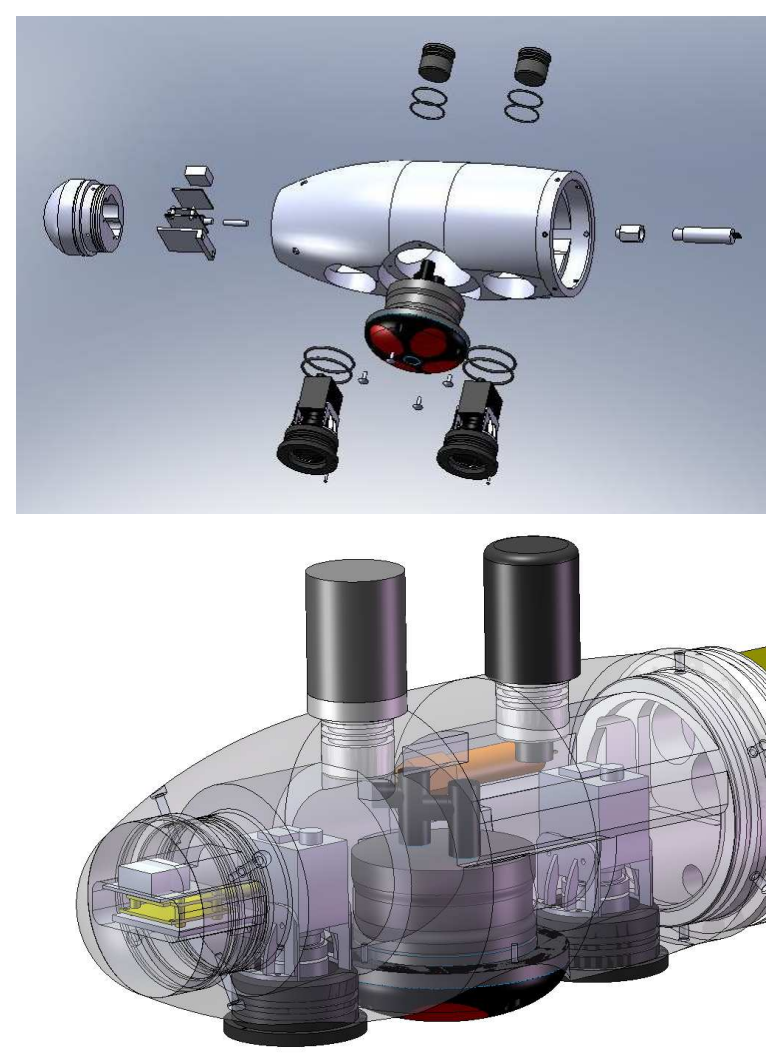

Fig. 6. Exploded and translucent view of PeRL's redesigned nose cone.

environment through a $1 / 8$ " shaft drilled perpendicular to the nose cone wall to reduce flow noise influence on the sensor. The Microstrain 3D-GX1 is integrated into the nose cone tip by mounting the Ocean-Server OS5000 Compass on top of the 3D-GX1 and milling a cavity in the tip to allow for the additional vertical clearance.

All o-rings installed in the nose cone are of material Buna-N (acrylonitrile-butadiene) and are lightly lubricated with Dow Corning \#4 prior to installation.

\section{Missions And TESTING}

Current missions and testing conducted by PeRL include testing at the UMich Marine Hydrodynamics Laboratory tow tank, automated visual ship hull inspection (conducted at AUVFest'08), field testing and engineering education demonstrations at the University of Michigan Biological Station (UMBS), and archaeological surveys of shipwrecks in the Thunder Bay National Marine Sanctuary (TBNMS).

\section{A. University of Michigan Marine Hydrodynamics Laboratory}

Initial in-water testing of the vehicle was held at the UMich physical model basin—a $109.7 \mathrm{~m}$ x $6.7 \mathrm{~m}$ x $3.0 \mathrm{~m}$ freshwater tow tank (Fig. 8). The MHL provides a controlled experimental setup for testing real-time visual SLAM systems in an aqueous environment. Prescribed vehicle motions can be precisely achieved via the electronically controlled tank carriage while imagery of the tank floor can be processed 

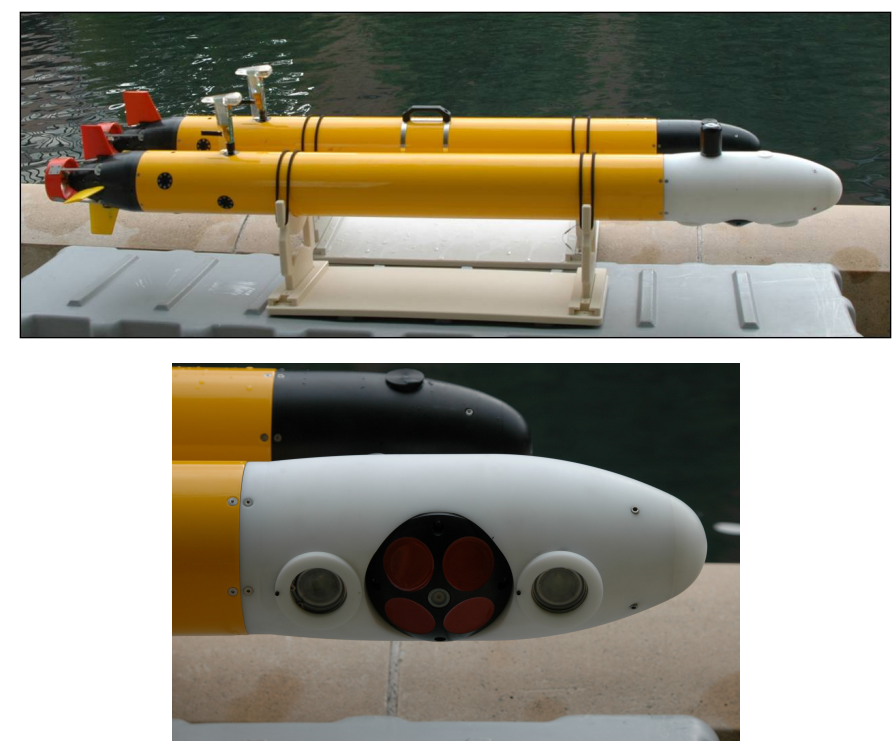

Fig. 7. (top) Modified Iver2 with new SLAM nose cone shown alongside a stock Iver2 vehicle. (bottom) A close up view of the SLAM nose cone with DVL and camera plugs.

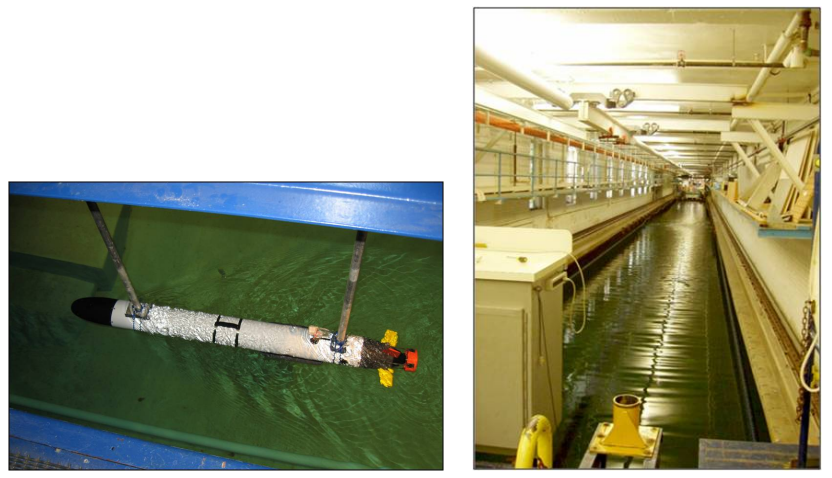

Fig. 8. Vehicle testing at the MHL tow tank.

online for visual SLAM estimation. For ease of real-time algorithm development we can attach a wet-mateable wired ethernet connection to a vehicle bulkhead so that imagery and sensor data can be streamed to a topside desktop computer allowing for greater flexibility in real-time software execution, visualization, and debugging. Use of the test facility has been beneficial for rapid prototyping, development, and validation of our real-time underwater visual SLAM hardware and algorithms.

\section{B. AUVFest'08}

The Iver2 testbed serves as a proxy for real-time visual autonomous port and hull inspection research at UMich where it is used to collect and perform visual SLAM navigation over the seafloor. To test our FBN algorithms in a real-hull environment, PeRL collaborated with Massachusetts Institute of Technology (MIT) and Bluefin Robotics to put one of our camera systems on the Hovering Autonomous Underwater Vehicle (HAUV) [21] at AUVFest'08 to collect imagery of the hull of the USS Saratoga-a decommissioned U.S. aircraft carrier stationed as Newport, RI (Fig. 9). PeRL packaged and mounted a calibrated Prosilica GC1380HC camera (same as that used in the Iver2 SLAM nose cone) and Deep Sea Power and Light (DSPL) light system on the HAUV hull inspection vehicle. Boustraphedeon survey imagery was collected by the HAUV of the hull of the USS Saratoga. The HAUV is equipped with a $1200 \mathrm{kHz}$ DVL, fiber optic gyro (FOG), and depth sensor, and is comparable to the sensor suite integrated into PeRL's Iver2 testbed.

Preliminary results for visual hull-relative navigation are shown in Fig. 9(d). Here we see a pose-graph of camera constraints generated through pairwise registration of overlapping imagery. These constraints are fused with navigation data in an extended information filter (EIF) [2] framework providing bounded error precision navigation along the hull. Each node in the network corresponds to a digital-still image taken along the hull (1033 images in all). Note the discrete dropout of images along the second leg in the region of $-10 \mathrm{~m}$ to $-20 \mathrm{~m}$ along the hull axis. Due to a logging error, we did not record any imagery during this time period; however, this gap in the visual data record actually highlights the utility of our hullreferenced visual navigation approach. Because we are able to pairwise register views of the hull taken from different times and locations, the camera process is able to "close-theloop" and register itself to earlier imagery from the first leg of the survey thereby reseting any incurred DVL navigation error during the data dropout period. It is precisely this hull-referenced navigation capability that allows the AUV to navigate in-situ along the hull without the need for deploying any external aiding (e.g. acoustic navigation transponders).

\section{University of Michigan Biological Station}

Field trials were held on Douglas Lake at the University of Michigan Biological Station (UMBS) in Pellston, Michigan during July, 2008. Four days of on-water testing demonstrated maneuverability, vehicle speed, dead-reckon navigation, wireless ethernet communication, sidescan sonar functionality, digital compass, and manual surface joystick operation modes. In addition to vehicle trials, PeRL staff presented guest lectures on AUVs and hosted hands-on training in conducting actual AUV missions. Launch and recovery were conducted from shore, dock, and from a pontoon boat. A full sidescan sonar survey of the south-eastern bay at Douglas Lake was run from the UMBS docks (Fig. 10). After the completion of the mission, the vehicle was manually motored, from a portable wireless station on the dock, back for recovery.

\section{Thunder Bay National Marine Sanctuary}

In August, 2008, PeRL collaborated with National Oceanic and Atmospheric Administration (NOAA) TBNMS researchers to map unexplored areas outside the Sanctuary's current boundaries. Established in 2000, the TBNMS protects one of the nation's most historically significant collections of shipwrecks. Located in the northeast corner of Michigan's lower peninsula, the 448 square-mile sanctuary contains 40 known historic shipwrecks. Archival research indicates that 


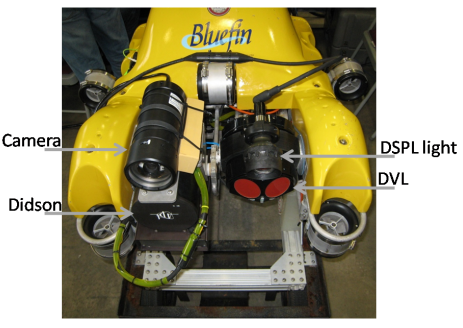

(a) HAUV

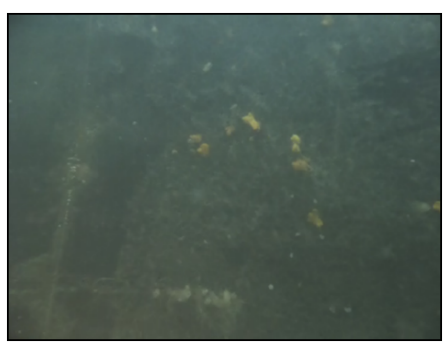

(b) Hull imagery

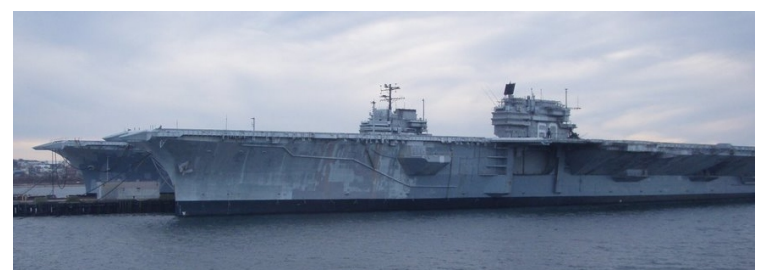

(c) USS Saratoga

Estimated Camera Trajectory 3-sigma

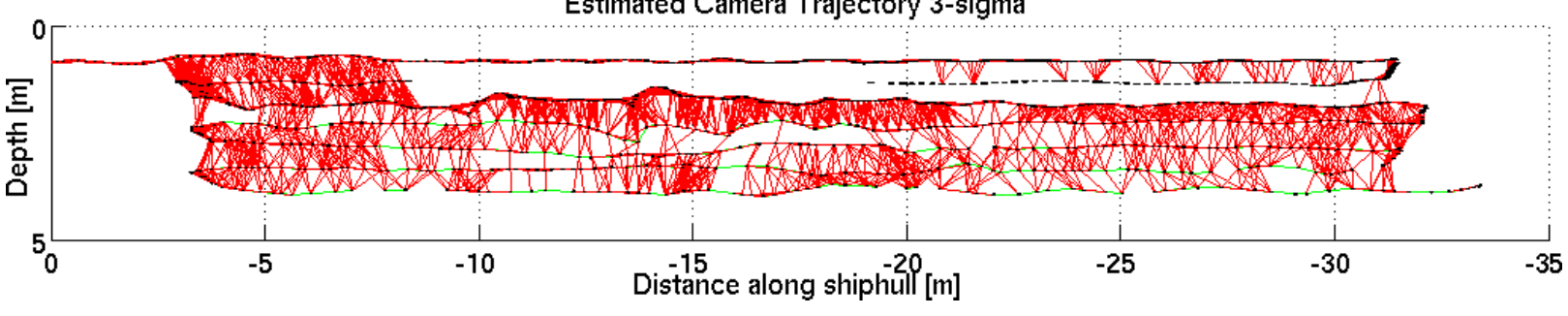

(d) VAN hull-referenced trajectory estimate.

Fig. 9. Vision-based autonomous hull mapping results for data collected at AUVFest'08 of the hull of the USS Saratoga.

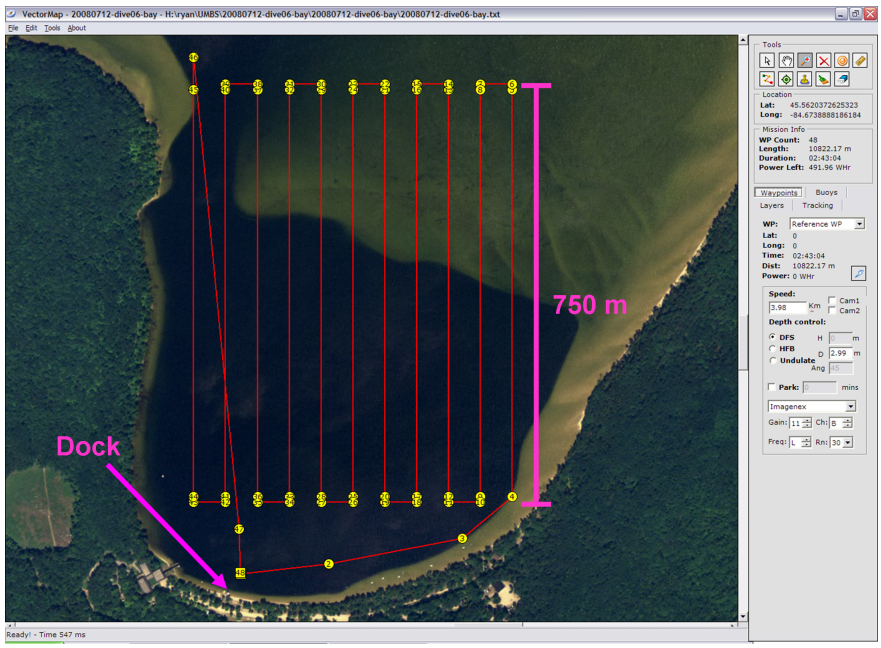

Fig. 10. Field testing at UMBS. The vehicle was launched and recovered from a dock on shore. Shown is a screenshot of Ocean-Server's Vectormap GIS mission planning interface.

over 100 sites await discovery within and just beyond the sanctuary's current boundaries. This fact, coupled with strong public support and the occurrence of dozens of known shipwrecks, provide the rationale for the sanctuary's desire to expand from 448 square-miles to 3,662 square-miles (an eight fold increase). To date, however, a comprehensive remote sensing survey has not been conducted in the potential expansion area. Moreover, significant portions of the existing sanctuary have not been explored. The potential for new discoveries in these areas is high.

PeRL is engaged in a five-year collaboration with TBNMS to use the Sanctuary as a real-world engineering testbed for our AUV algorithms research. TBNMS provides in-kind support of ship time and facilities, and in return receives SLAM-derived archaeological data products ranging from 3D photomosaics of ship wrecks to sidescan sonar maps of the Sanctuary seafloor. In addition, PeRL is engaged in public outreach efforts in collaboration with TBNMS to educate the general public in the use and technology of underwater robotics. In development is an AUV technology display in their stateof-the-art Great Lakes Maritime Heritage Center, (a 20,000 square foot building featuring a 100-seat theater, 9,000 square feet of exhibit space, and distance learning capabilities) that will consist of an Iver2 AUV hull, a multimedia kiosk, and maps and data products derived from PeRL's field testing in the Sanctuary.

This past August, as part of a NOAA Ocean Exploration grant, PeRL fielded one of its two Iver2 AUVs to collect sidescan sonar imagery in unmapped regions of the Sanctuary seafloor. Of the two Iver2 vehicles owned by PeRL, this vehicle had not yet been modified with the new SLAM nose cone and additional sensors. However, it still presented an opportunity to get practice with the operational side of vehicle launch, recovery, in-water tracking, and to test the sidescan sonar system. Fig. 11 shows survey tracklines and sonar imagery collected of a newly found wreck outside of the Sanctuary's boundaries in approximately $50 \mathrm{~m}$ of water depth.

\section{CONCLUSiON}

This paper provided an overview of PeRL's AUV algorithms research and testbed development at the University of Michigan. To summarize, PeRL's main research thrusts are in the areas of (1) real-time visual SLAM; (2) cooperative multi-vehicle navigation; and (3) perception-driven control. Toward that goal we reported the modifications involved in preparing two commercial Ocean-Server AUV systems for 

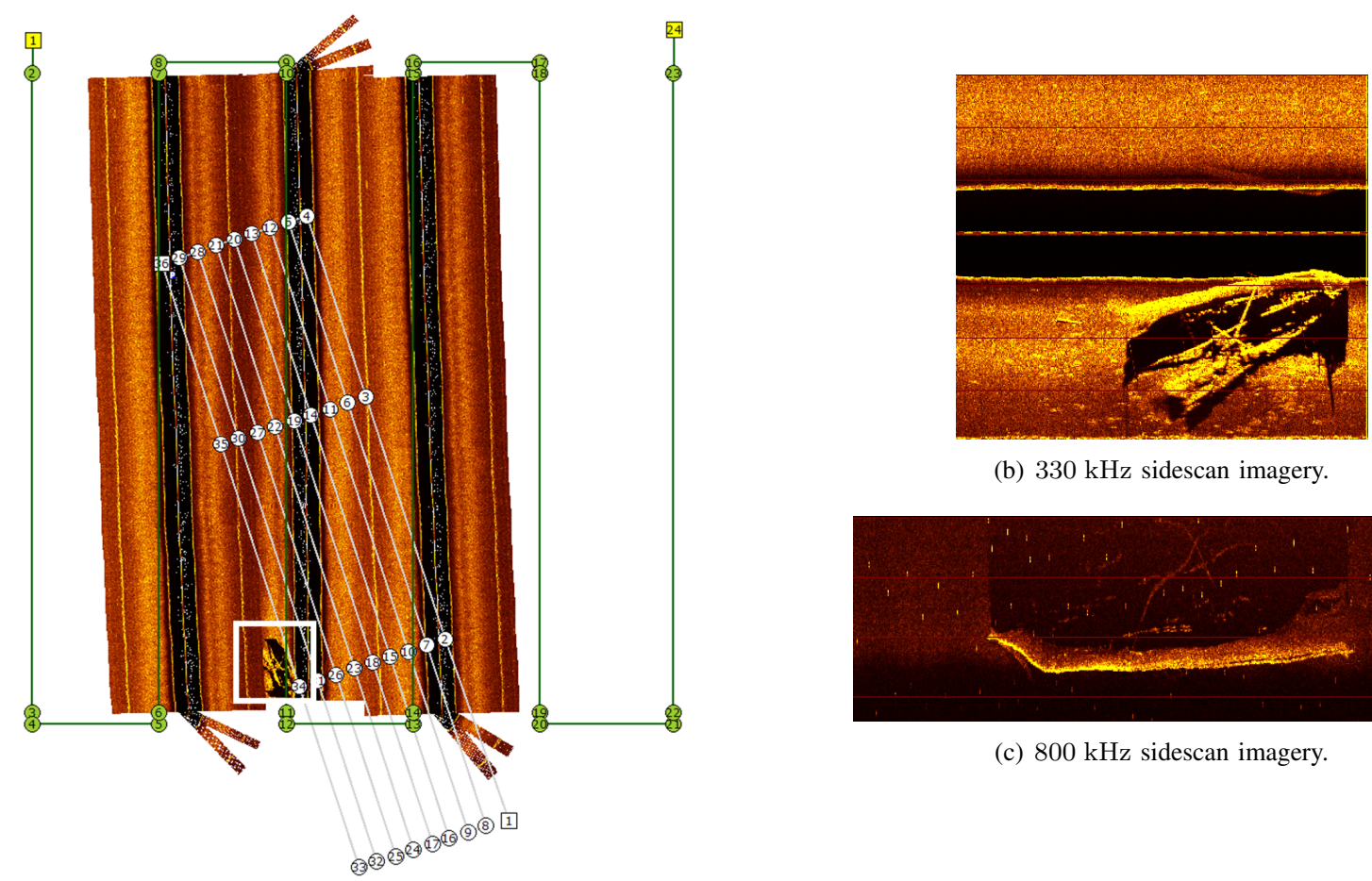

(b) $330 \mathrm{kHz}$ sidescan imagery.

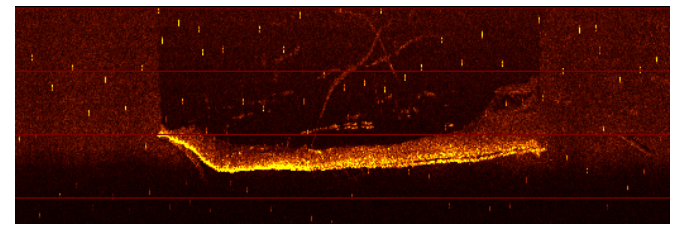

(c) $800 \mathrm{kHz}$ sidescan imagery.

(a) Mission overlay.

Fig. 11. Sidescan sonar mapping results from testing this summer in TBNMS. (a) A large area search was conducted first to locate the target wreck indicated in the white box (survey tracklines are shown in green). A second finer-scale survey was then conducted to map the target at higher resolution (tracklines overlaid in gray). (b) Target imagery found using $330 \mathrm{kHz}$ sonar. (c) Detailed target imagery using $800 \mathrm{kHz}$ sonar.

SLAM research at the University of Michigan. PeRL upgraded the vehicles with additional navigation and perceptual sensors including 12-bit stereo down-looking Prosilica cameras, a Teledyne $600 \mathrm{kHz}$ RDI Explorer DVL for 3-axis bottom-lock velocity measurements, a KVH single-axis fiber-optic gyroscope for yaw rate, and a WHOI Micromodem for communication, along with other sensor packages. To accommodate the additional sensor payload, a new Delrin nose cone was designed and fabricated. Additional 32-bit embedded CPU hardware was added for data-logging, real-time control, and in-situ real-time SLAM algorithm testing and validation.

The impact of this research will be new multi-AUV navigation performance that will enable new missions for ocean science presently considered impractical or infeasible. The fundamental robotic navigation algorithms developed in this body of work will provide the requisite spatial scale, duration, and autonomy required for in-situ benthic exploration by AUVs. Our field testing and collaboration with the TBNMS will provide validation of the proposed navigation methodologies in a real-world engineering testbed, while a new museum exhibit on underwater robotics at their visitor center will disseminate the findings and results of this research to the general public.

\section{ACKNOWLEDGMENT}

This work is supported in part through grants from the National Science Foundation (NSF) (Award \#IIS 0746455), the Office of Naval Research (ONR) (Award \#N00014-07-
1-0791), and a NOAA Ocean Exploration grant (Award \#WC133C08SE4089).

\section{REFERENCES}

[1] R. M. Eustice, O. Pizarro, and H. Singh, "Visually augmented navigation in an unstructured environment using a delayed state history," in Proc. IEEE Intl. Conf. Robot. Auto., vol. 1, New Orleans, USA, Apr. 2004, pp. 25-32.

[2] R. M. Eustice, H. Singh, and J. J. Leonard, "Exactly sparse delayedstate filters for view-based SLAM," IEEE Trans. Robot., vol. 22, no. 6, pp. 1100-1114, Dec. 2006, king-Sun Fu Memorial Best Transactions on Robotics Paper Award of the IEEE Robotics and Automation Society.

[3] R. M. Eustice, H. Singh, J. J. Leonard, and M. R. Walter, "Visually mapping the RMS Titanic: conservative covariance estimates for SLAM information filters," Intl. J. Robotics Research, vol. 25, no. 12, pp. 12231242, 2006.

[4] R. M. Eustice, O. Pizarro, and H. Singh, "Visually augmented navigation for autonomous underwater vehicles," IEEE J. Oceanic Eng., vol. 33, no. 2, 2008.

[5] R. M. Eustice, "Toward real-time visually augmented navigation for autonomous search and inspection of ship hulls and port facilities," in Intl. Symposium on Technology and the Mine Problem, Monterey, CA, May 2008.

[6] B. Foley, K. DellaPorta, D. Sakellariou, B. Bingham, R. Camilli, R. Eustice, D. Evagelistis, V. Ferrini, M. Hansson, K. Katsaros, D. Kourkoumelis, A. Mallios, P. Micha, D. Mindell, C. Roman, H. Singh, D. Switzer, and T. Theodoulou, "New methods for underwater archaeology: the 2005 Chios ancient shipwreck survey," Hesperia, Accepted, To Appear.

[7] P. Milne, Underwater acoustic positioning systems. Houston: Gulf Publishing Company, 1983.

[8] M. Hunt, W. Marquet, D. Moller, K. Peal, W. Smith, and R. Spindel, "An acoustic navigation system," Woods Hole Oceanographic Institution, Tech. Rep. WHOI-74-6, Dec. 1974. 
[9] R. M. Eustice, L. L. Whitcomb, H. Singh, and M. Grund, "Recent advances in synchronous-clock one-way-travel-time acoustic navigation," in Proc. IEEE/MTS OCEANS Conf. Exhib., Boston, MA, USA, Sep. 2006, pp. $1-6$.

[10] M. R. Walter and J. J. Leonard, "An experimental investigation of cooperative SLAM," in Proc. IFAC/EURON Symp. Intell. Autonomous Vehicles, Lisbon, Portugal, Jul. 2004.

[11] I. Rekleitis, G. Dudek, and E. Milios, "Probabilistic cooperative localization and mapping in practice," in Proc. IEEE Intl. Conf. Robot. Auto., vol. 2, Sep. 2003, pp. 1907-1912.

[12] F. Bourgault, T. Furukawa, and H. Durrant-Whyte, "Decentralized Bayesian negotiation for cooperative search," in Proc. IEEE/RSJ Intl. Conf. Intell. Robots Systems, vol. 3, Sep. 2004, pp. 2681-2686.

[13] S. B. Williams, G. Dissanayake, and H. Durrant-Whyte, "Towards multivehicle simultaneous localisation and mapping," in Proc. IEEE Intl. Conf. Robot. Auto., vol. 3, Washington, DC, May 2002, pp. 2743-2748.

[14] M. Ridley, E. Nettleton, S. Sukkarieh, and H. Durrant-Whyte, "Tracking in decentralised air-ground sensing networks," in Proc. IEEE Intl. Conf. Information Fusion, vol. 1, Jul. 2002, pp. 616-623.

[15] L.-L. Ong, B. Upcroft, T. Bailey, M. Ridley, S. Sukkarieh, and H. Durrant-Whyte, "A decentralised particle filtering algorithm for multitarget tracking across multiple flight vehicles," in Proc. IEEE/RSJ Intl. Conf. Intell. Robots Systems, Beijing, Oct. 2006, pp. 4539-4544.
[16] J. Partan, J. Kurose, and B. N. Levine, "A survey of practical issues in underwater networks," in Proc. ACM Intl. Workshop on Underwater networks. New York, NY, USA: ACM Press, 2006, pp. 17-24.

[17] R. M. Eustice, L. L. Whitcomb, H. Singh, and M. Grund, "Experimental results in synchronous-clock one-way-travel-time acoustic navigation for autonomous underwater vehicles," in Proc. IEEE Intl. Conf. Robot. Auto., Rome, Italy, Apr. 2007, pp. 4257-4264.

[18] L. Freitag, M. Grund, S. Singh, J. Partan, P. Koski, and K. Ball, "The WHOI micro-modem: an acoustic communications and navigation system for multiple platforms," in Proc. IEEE/MTS OCEANS Conf. Exhib., Washington, D.C., Sep. 2005, pp. 1086-1092.

[19] L. Freitag, M. Grund, J. Partan, K. Ball, S. Singh, and P. Koski, "Multiband acoustic modem for the communications and navigation aid AUV," in Proc. IEEE/MTS OCEANS Conf. Exhib., Washington, D.C., Sep. 2005, pp. 1080-1085.

[20] B. Anderson and J. Crowell, "Workhorse AUV - a cost-sensible new autonomous underwater vehicle for surveys/soundings, search \& rescue, and research," in Proc. IEEE/MTS OCEANS Conf. Exhib., Sep. 2005 , pp. $1228-1233$

[21] J. Vaganay, M. Elkins, S. Willcox, F. Hover, R. Damus, S. Desset, J. Morash, and V. Polidoro, "Ship hull inspection by hull-relative navigation and control," in Proc. IEEE/MTS OCEANS Conf. Exhib., 2005, pp. 761-766. 